\title{
Understanding the Role of Acid vs. Electron Blur in EUV Resist Materials
}

\author{
James W. Thackeray ${ }^{1}$, Mike Wagner ${ }^{1}$, Su Jin Kang ${ }^{1}$ and John Biafore ${ }^{2}$ \\ ${ }^{1}$ Dow Electronic Materials \\ 455 Forest St Marlboro MA 01752-4650 \\ ${ }^{2}$ KLA-Tencor, PCID division \\ 8843 N. Capital of Texas Highway Austin, TX 78759
}

\begin{abstract}
Further development of a polymer-bound PAG resist system is discussed. For $28 \mathrm{~nm}$ hp, we obtained 26\% exposure latitude and 0.3 micron DOF at a sensitivity of $17.1 \mathrm{mj}$. We also obtained $20 \mathrm{~nm}$ half pitch resolution under dipole conditions. We have undertaken to deconvolve the blur sources in EUV as well. The total diffusion blur of an EUV resist exposed under ArF exposure conditions was estimated to be $10.6 \mathrm{~nm}$, with $9.3 \mathrm{~nm}$ of the blur coming from acid reaction-diffusion and $4.3 \mathrm{~nm}$ coming from the radius of gyration of the polymer. The total diffusion blur of the same resist material under EUV exposure conditions is $11.5 \mathrm{~nm}$. We get an EUV-specific blur contribution of $2.5 \mathrm{~nm}$ associated with the acid cloud generated from the electrons generated after an EUV photon hits the polymer matrix, and a presently undefined blur contribution of $3.7 \mathrm{~nm}$ which is probably contributed from the flare of the optics, and possibly from out-of-band radiation. The polymer-bound PAG resist has effectively a $9.7 \mathrm{~nm}$ diffusion blur contribution which should be improved to less than $8 \mathrm{~nm}$ for $16 \mathrm{~nm}$ node.
\end{abstract}

Keywords: EUV resist, diffusion blur, Polymer-bound PAG

\section{Introduction}

EUV lithography presents a new paradigm in the nature of the EUV radiation and how it interacts with the resist material to form the acid latent image necessary for pattern formation. $[1,2]$ We are particularly interested in the blurring phenomena that control the contrast of information deposited in the resist and the consequences of the physico-chemical changes that the exposed resist is subject to during standard lithographic processing. $[3,4,5]$ Figure 1 shows a typical EUV exposure process and the theoretical resist interaction with EUV radiation. The formation of a resist relief image at EUV may be divided into steps:

1. generation of the projection image

2. the absorption of an EUV photon by the resist
3. ionization and generation ofphotoelectrons by the resist

4. the scattering of low-energy electrons that may convert PAG to acid

5. reaction and diffusion of the acid during PEB to deprotect the matrix polymer.

This complex process is not well understood and leads to challenges and opportunities in EUV resist design. In particular, we are most interested in studying how the various sources of image blur in an EUV process are partitioned. In this paper, we have exposed a polymer-bound PAG resist system [6,7] under EUV and ArF exposure conditions and used stochastic resist simulation to attempt to differentiate the role of ionizing vs. non-ionizing radiation on image blur. It is thought that in $\mathrm{ArF}$ exposure, the resist is not ionized and 
that acid generation primarily occurs by direct photolysis of the acid generator. [8] By exposing the same resist at EUV and ArF, we can study the events unique to ArF exposure vs. EUV exposure which produce image blur. [5]

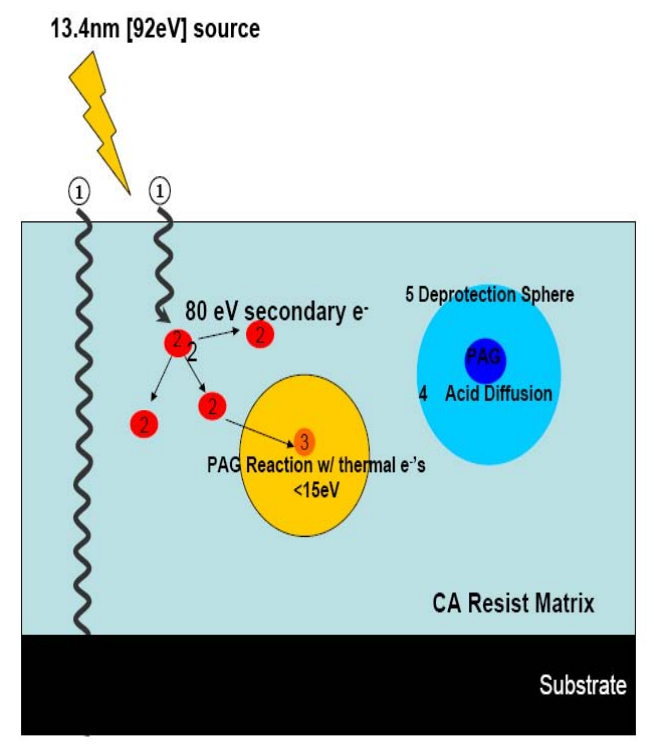

Figure 1. Interaction of EUV radiation with conventional Chemically Amplified Resist Material.

\section{Experimental:}

2.1. Resist materials.

All new materials were formulated in 80/20 blend of ethyl lactate (EL) and propylene glycol monomethyl ether acetate (PGMEA). We formulated an EUV and ArF sensitive resist material called EUV Resist A as outlined in Figure 2. For these experiments we used a traditional ArF low activation leaving group [LG], and an EUV sensitization monomer to enhance secondary electron yield. Also we used a triphenyl sulfonium PAG which has good sensitivity in the EUV and ArF exposure modes. The PAG anion is bound to the polymer to reduce acid diffusion length. Traditional quencher was used as needed.

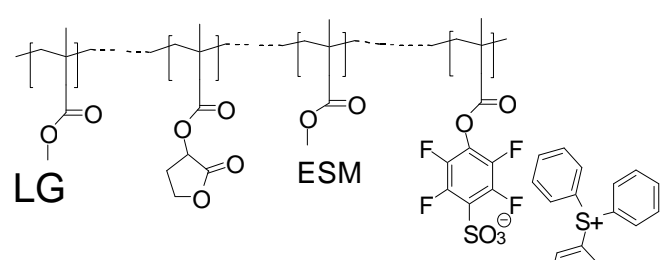

Figure 2. Prototypical EUV polymer used in EUV Resist A for these experiments.

\subsection{Resist Processing}

The formulated resists were spin coated onto $25 \mathrm{~nm}$ AR107 BARC coated silicon wafer. The latter undercoat was used for the sole purpose of promoting adhesion. The resist film thickness was $40 \mathrm{~nm}$ for EUV exposures and 70nm for $\mathrm{ArF}$ exposures. All resists were soft baked at $140{ }^{\circ} \mathrm{C}$ for 60 seconds. The post exposure bake (PEB) was varied from $90{ }^{\circ} \mathrm{C}$ to $110{ }^{\circ} \mathrm{C}$. The resists were initially screened for clearing dose $\left(\mathrm{E}_{0}\right)$, thinning and contrast curves using ArF exposure at Rohm and Haas FAB facilities in Marlborough. The ArF scanner is an ASM-L 11000.75 NA. Some of the EUV exposures were conducted at Lawrence Berkeley National Laboratory (LBNL) on MET ALS EUV tool. The Berkeley MET EUV tool possesses an NA of 0.30 and was operated on $\mathrm{Y}$ monopole. Most of the EUV evaluation data came from Albany SEMATECH EMET tool with an NA of 0.30 , quadrupole conditions.

\section{Results}

3.1 ArF results with polymer-bound PAG resist.

This resist was tested in ArF on an ASM-L 1100 scanner. The resist was processed in the identical manner as would be done with EUV exposure except the resist thickness was 30nm thicker at $70 \mathrm{~nm}$ film thickness and the organic antireflective stack was optimized for zero reflectivity from the Si substrate. The CDs studied were $75 \mathrm{~nm}$ half-pitch using dipole illumination, a rather challenging feature for a $0.75 \mathrm{NA}$ scanner. Figure 3 shows top-down SEM images of the $75 \mathrm{~nm}$ CD.

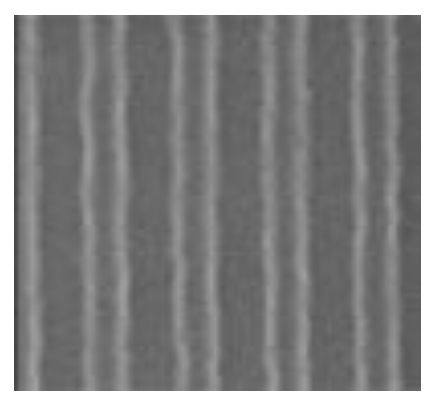

Figure 3. $75 \mathrm{~nm} \mathrm{hp} \mathrm{images} \mathrm{in} \mathrm{an} \mathrm{EUV} \mathrm{resist} \mathrm{A} \mathrm{exposed}$ on an ArF scanner. Dose to size is $10 \mathrm{~mJ}$.

Figure 4 shows the exposure latitude for the same resist. The exposure latitude for the resist is $16 \%$ for a $+/-10 \% \mathrm{CD}$ change, or alternatively, 
$5.6 \mathrm{~nm} / \mathrm{mj}$. Loss of exposure latitude in real CA resists from theoretical NILS can be used as a measure of acid diffusion blur and will be discussed later.

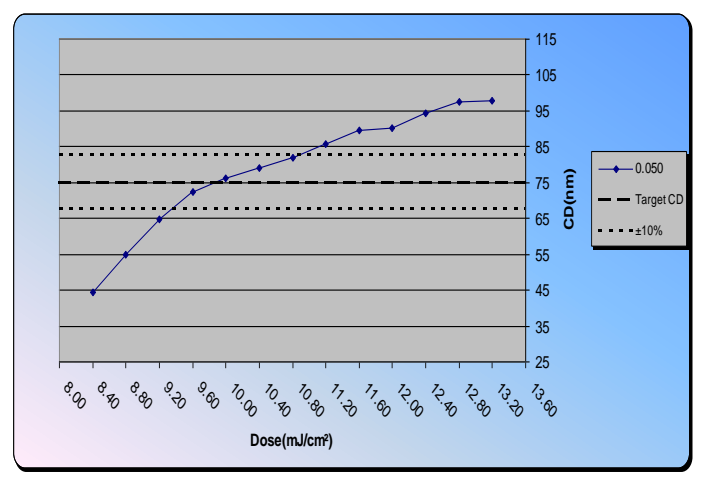

Figure 4. ArF exposed EUV resist A showing exposure latitude for $75 \mathrm{~nm} \mathrm{hp} \mathrm{CD}$. Exposure latitude is $16 \%$ for a $+/-10 \%$ CD change.

\subsection{EUV exposure results.}

EUV resist A was exposed on the Albany EMET EUV exposure tool under quadrupole conditions. Figure 5 shows the $28 \mathrm{~nm}$ hp process window obtained for the resist. The calculated exposure latitude for this resist under EUV conditions is $26 \%$ for a $+/-10 \%$ CD change, or alternatively, $0.30 \mathrm{~nm} / \mathrm{mJ}$. We can also use the experimental exposure latitude vs NILS to calculate diffusion blur. In the EUV case, there may be an added contribution of the diffusion of secondary electron blur which is not possible in ArF exposure. Figure 6 shows the ultimate resolution ability of the polymer-bound PAG resist under dipole conditions using the EMET tool. This figure shows $20 \mathrm{~nm}$ hp capability at a photospeed less than $20 \mathrm{~mJ}$.

\section{Stochastic Resist Simulation and Modeling}

Acid generation in chemically-amplified resists first requires the absorption of energy in the form of light. The initiating activation of a photoreaction is provided by the absorption of light leading to an electronically excited state; the thermodynamicallyfavorable products which are accessible to an electronically excited molecule are greater than those accessible to a ground-state molecule, since the excited molecule possesses excess energy as a result of photon absorption. [8] The chronology of photoreactions can be divided into three stages: photon absorption and formation of the excited

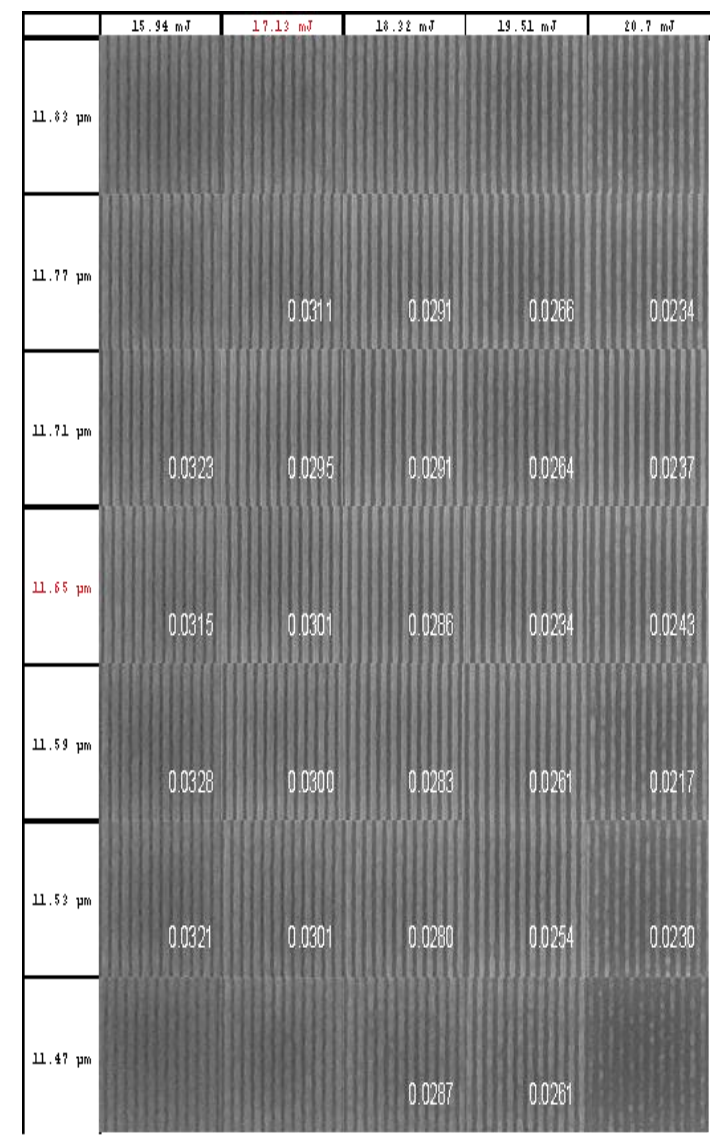

Figure 5. $28 \mathrm{~nm} \mathrm{hp} \mathrm{process} \mathrm{window} \mathrm{for} \mathrm{EUV} \mathrm{resist} \mathrm{A}$ exposed on the Albany EMET exposure tool.

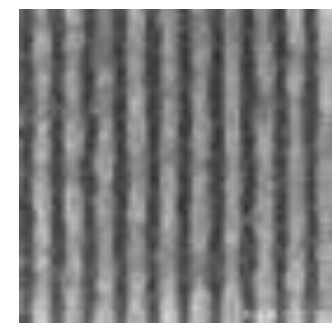

Figure 6. 20nm hp resolution for EUV resist A exposed under dipole conditions

state, the primary or direct photochemical process, the secondary or indirect process which occurs from the intermediates produced during the primary process.

Let $\mathrm{n}$ represent the total number of photons absorbed by an irradiated volume of resist. The average number of photons absorbed by the volume of resist is: 


$$
E(n)=\langle n\rangle=\alpha \cdot \operatorname{dos} e \cdot V \cdot(h v)^{-1}
$$

where $\alpha$ is the absorbance coefficient in units of $1 / \mathrm{m}$ and hv is the photon energy in Joules. As the energy of the photon increases, the number of absorbed photons decreases and the total absorbed energy is discretized among fewer quanta. The distribution of $\mathrm{n}$ is Poisson, accordingly

$$
\operatorname{Var}(n)=\langle n\rangle
$$

The relative uncertainty in receiving the expected number of absorbed photons is the relative photon shot noise (RPSN) and is equal to the ratio of the standard deviation of $n$ to the expectation of $n$

$$
R P S N=\frac{\sqrt{\operatorname{Var}(n)}}{E(n)}=\frac{\sqrt{\langle n\rangle}}{\langle n\rangle}=\frac{1}{\sqrt{\langle n\rangle}}
$$

Equation 3 describes the phenomenon of photon shot noise and shows that the uncertainty in absorbing the expected number of photons is inversely proportional to the square-root of the average number of absorbed photons. Shot noise is more problematic at EUV, where many fewer photons are absorbed compared to $\operatorname{ArF}$ at identical dose. In order to speed wafer processing, the targeted resist sizing dose at EUV is about $10 \mathrm{~mJ} / \mathrm{cm}^{2}$. To illustrate, in a contact hole $30 \mathrm{~nm}$ x by $30 \mathrm{~nm}$ y by $100 \mathrm{~nm} \mathrm{z}$ with resist absorbance $4 / \mathrm{um}$, at 10 $\mathrm{mJ} / \mathrm{cm}^{2}$, about 2626 photons are absorbed at $\mathrm{ArF}$, while only 526 photons are absorbed at EUV. The relative photon shot noise is $1.9 \%$ at $\mathrm{ArF}$ and $4.4 \%$ at EUV.

In the soft $\mathrm{x}$-ray region $(0.09-2.5 \mathrm{keV}, 13.5$ $\mathrm{nm}=0.092 \mathrm{keV})$, the absorption of a photon can result in ionization of the resist and the ejection of a photoelectron with excess kinetic energy. [9] The maximum kinetic energy of the photoelectron can be estimated by knowledge of the ionization potential of the material. If the photon energy $h v$ is greater than the ionization potential IP, a photoelectron with maximum kinetic energy KE may be dislodged from the absorbing atom or molecule.
(4)

$$
K E_{\max }=\frac{1}{2} m_{e} v^{2}=h \frac{c}{\lambda}-I P
$$

where $m_{e}$ is the rest mass of an electron, $v$ is the velocity of the electron, $\mathrm{hc} / \lambda$ is the photon energy. Though the absorption is localized at a given atomic site, the photoelectron and any secondary electrons generated by further ionization of the resist matrix are thought to redistribute the energy of the incident photon spatially, an effect known as photoelectron blur. [10]

At EUV, acid generators are thought to be activated primarily by scattered low-energy electrons, [11] suggesting that acids may be formed a distance from the absorption site, in effect reducing contrast. Figure 7 shows a plot of the positions of acids produced by 5000 independent photon absorption events using a discrete probabilistic ionization and electron scattering model. Acids are generated by scattered low-energy electrons. In the simulation, ionization potential of the resist is $10 \mathrm{eV}$, PAG reaction radius is $2 \mathrm{~nm}$, and minimum excitation energy for PAGs is $5 \mathrm{eV}$. The average radius of the acid distribution in this example is about $4 \mathrm{~nm}$. At ArF, photon energy is about $6.4 \mathrm{eV}$, probably less than the resist ionization potential. Accordingly, the acid generation mechanism at ArF is thought to occur by the direct photolysis of the acid generator.

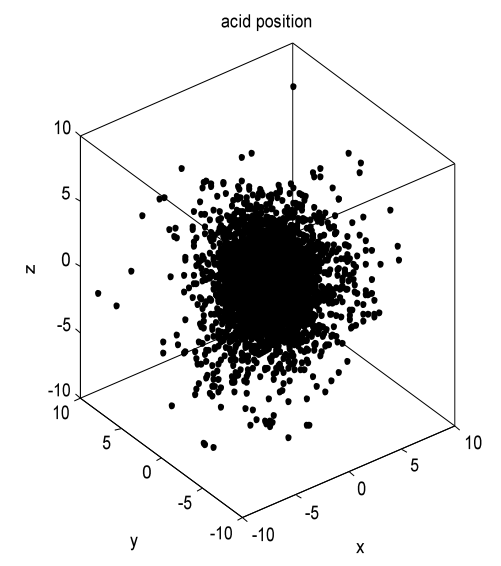

Figure 7. Simulated discrete acid positions produced by 1000 photon absorption events at a common origin (with replacement, e.g. each trial is independent).

Using stochastic resist simulation, we attempt to solve, by iterative improvement of the solution, for a set of unknown physical simulation parameters that can reasonably describe 
lithographic properties of a polymer-bound resist exposed at EUV and ArF. For the purpose of this study, it's assumed that the ArF projection optics have negligible flare and aberrations (ASML /1100). The reaction-diffusion and development properties of the resist are assumed to be unaffected by processing at either EUV or ArF. A single set of physical parameters describing reaction-diffusion and development are applied to all data. Figure 8 shows a comparison of stochastic simulation vs. data at EUV; Figure 8 shows a comparison of stochastic simulation vs. data at ArF.
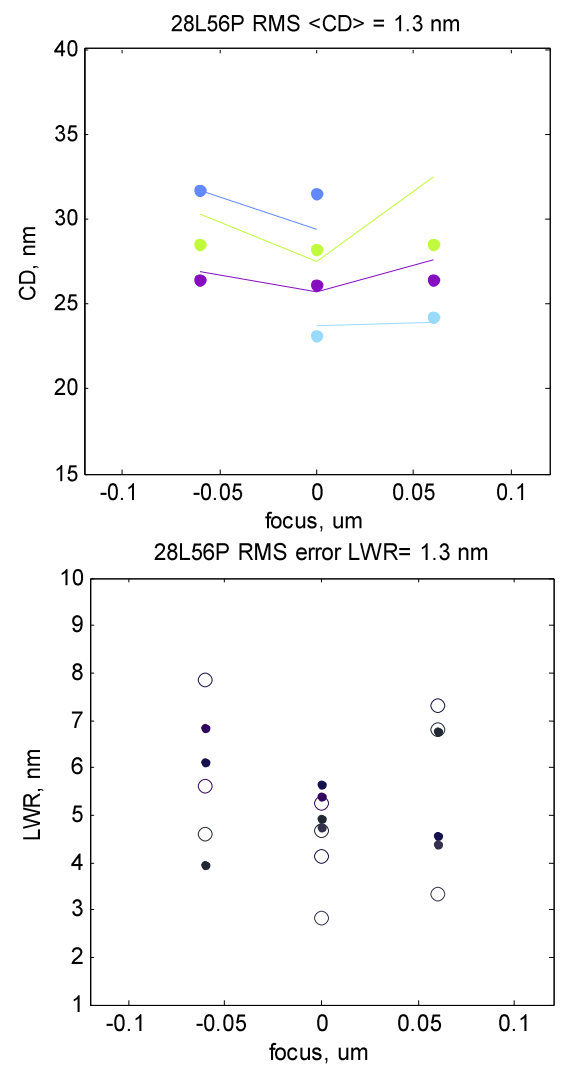

Figure 8. A comparison of stochastic simulation vs. data at EUV using $0.30 \mathrm{NA}$, quadrupole, 0.22 center sigma, 0.11 radial sigma. The plot to left shows model fit to experimental $\langle\mathrm{CD}\rangle$ vs. focus and dose for $28 \mathrm{~nm}$ lines on $56 \mathrm{~nm}$ pitch. Dots represent data, lines represent simulations. The plot to right shows model fit to LWR for $28 \mathrm{~nm}$ lines on $56 \mathrm{~nm}$ pitch; circles represent simulation, dots represent data.

The unknown parameter values of the physical simulator have been converged to (simultaneously) average CD and LWR for $28 \mathrm{~nm}$ lines on $56 \mathrm{~nm}$ pitch vs. dose and focus at EUV and exposure

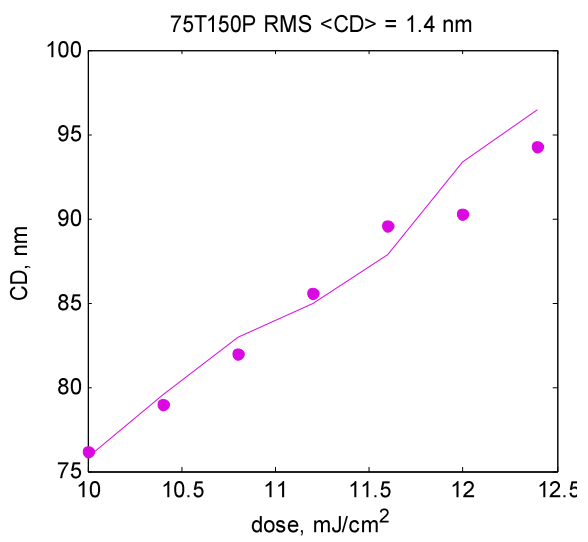

Figure 9. A comparison of stochastic simulation vs. data at ArF using 0.75 NA, dipole, 0.69 outer sigma, 0.39 inner. The plot shows model fit to experimental trench <CD> vs dose for $75 \mathrm{~nm}$ trenches on $150 \mathrm{~nm}$ pitch. Dots represent data, lines represent simulations.

latitude for $75 \mathrm{~nm}$ trenches on $150 \mathrm{~nm}$ pitch at ArF. Un-weighted simulation error is $1.3 \mathrm{~nm}$ RMS with maximum error of $3 \mathrm{~nm}$. The total blur contributed by the resist process can be estimated in a straightforward way by simulating the resist exposure latitude and comparing to data. Total blur contributed by the ArF resist process is determined to be about $10.6 \mathrm{~nm}$ in this case.

The contributions of the components of blur add in quadrature and may be estimated

$$
b=\left(\sum_{i} p_{i}^{2}\right)^{1 / 2}
$$

where $\mathrm{b}$ is the total blur in $\mathrm{nm}$ and $\mathrm{p}$ is the blur contributed by the ith process. Using (5), we determine that blur contributed by resist latent image reaction-diffusion during PEB is about 9.7 nm

(6)

$$
\begin{aligned}
& \left(L I^{2}+R O G^{2}\right)^{1 / 2}=\left(L I^{2}+(4.3 \mathrm{~nm})^{2}\right)^{1 / 2}=10.6 \mathrm{~nm} \\
& L I=9.7 \mathrm{~nm}
\end{aligned}
$$

where LI is the blur attributable to latent image reaction-diffusion during PEB and ROG is the radius-of-gyration.

It's interesting to note that summing the $1 \mathrm{~d}$ acid diffusion length and the radius-of-gyration in quadrature yield a blur length of $11.8 \mathrm{~nm}$. The 
difference between the two estimations, $9.7 \mathrm{~nm}$ vs. $11.8 \mathrm{~nm}$, may be attributed to quencher diffusion and neutralization of acid during PEB, unaccounted for in (6).

Total blur for EUV exposure attributed to the EUV resist is estimated to be $11.5 \mathrm{~nm}$. Using LI calculated above and ROG

$$
\left((9.7 \mathrm{~nm})^{2}+(4.3 \mathrm{~nm})^{2}+x^{2}\right)^{1 / 2}=11.5 \mathrm{~nm}
$$

$x=4.5 \mathrm{~nm}$

suggesting about $4.5 \mathrm{~nm}$ of the total blur is unaccounted for in the analysis.

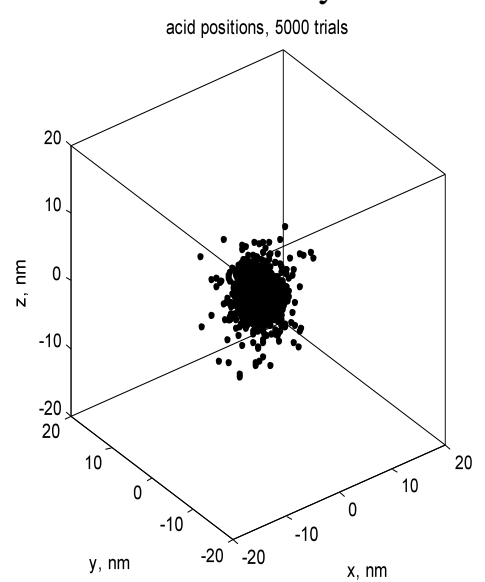

Figure 10. Simulated discrete acid positions produced by 5000 photon absorption events at a common origin.
We may use best-fit parameters to approximate the radius of the acid cloud produced by EUV exposure. Figure 10 shows a plot of the simulated discrete acid positions for 5000 independent photon absorption events at a common origin.

Acids are generated with replacement by scattered low-energy electrons. In the simulation, ionization potential of the resist is $9.75 \mathrm{eV}, \mathrm{PAG}$ reaction radius is $1.3 \mathrm{~nm}$, and minimum excitation energy for PAGs is $5.5 \mathrm{eV}$. The average radius of the simulated acid distribution is about $2.5 \mathrm{~nm}$. Revisiting (7) and representing the blur contributed by the acid distribution post-exposure as $\mathrm{E}$

$$
\left((9.7 \mathrm{~nm})^{2}+(4.3 \mathrm{~nm})^{2}+(2.5 \mathrm{~nm})^{2}+x^{2}\right)^{1 / 2}=11.5 \mathrm{~nm}
$$

$x=3.7 \mathrm{~nm}$

suggests that about $3.7 \mathrm{~nm}$, about $1 / 3$ of the total blur at EUV, is unaccounted for in the analysis.

\section{Summary}

The total diffusion blur of an EUV resist exposed under $\operatorname{ArF}$ exposure conditions was estimated to be $10.6 \mathrm{~nm}$, with $9.3 \mathrm{~nm}$ of the blur coming from acid reaction-diffusion and $4.3 \mathrm{~nm}$ coming from the radius of gyration of the polymer.

\begin{tabular}{|l|l|l|}
\hline Table of estimated simulation parameters & EUV & ArF \\
\hline Refractive index & $1.0-0.005 \mathrm{i}$ & $1.8-0.087 \mathrm{i}$ \\
\hline ( <n Quenchers/nm3 ) / ( <n PAGs>/nm3 ) & 0.09 & 0.09 \\
\hline PAG Quantum Efficiency & 0.19 & 0.19 \\
\hline Acid Diffusivity Rate (nm2/s) & 0.998 & 0.998 \\
\hline Quencher Diffusivity Rate (nm2/s) & 1.000 & 1.000 \\
\hline Amplification Reaction Rate (nm3/s) & 1.99 & 1.99 \\
\hline Acid-Quencher Neutralization Rate (nm3/s) & 25.00 & 25.00 \\
\hline Development Mth & 0.71 & 0.71 \\
\hline Development n & 20.29 & 20.29 \\
\hline Electron Generation Efficiency & 0.96 & 0 \\
\hline Ionization Potential (eV) & 9.75 & 9.75 \\
\hline Acid Generation Reaction Radius (nm) & 1.34 & 1.34 \\
\hline Acid Generator Excitation Energy (eV) & 5.48 & 5.48 \\
\hline Polymer Radius of Gyration (nm) & 4.3 & 4.3 \\
\hline
\end{tabular}

Table 1. Estimated values of stochastic simulation parameters for a polymer-bound PAG resist exposed at EUV and ArF. 
When we calculate the total diffusion blur of the same resist material under EUV exposure conditions the total diffusion blur increases to $11.5 \mathrm{~nm}$. Assuming that the blur contributions in ArF are identical in EUV, we can use the value for acid reaction-diffusion [9.3nm] and radius of gyration [4.3nm]. When we solve for the unaccounted for blur, we get an EUV-specific blur contribution of $2.5 \mathrm{~nm}$ associated with the acid cloud generated from the electrons generated after an EUV photon hits the polymer matrix, and a presently undefined blur contribution of $3.7 \mathrm{~nm}$ which may be attributed to any of several sources such as flare, mask roughness, out-of-band radiation, etc. The polymer-bound PAG resist has effectively a $9.7 \mathrm{~nm}$ diffusion blur contribution which should be improved to less than $8 \mathrm{~nm}$ for $16 \mathrm{~nm}$ node.

\section{References}

1. D. Attwood, "Soft X-rays and Extreme Ultraviolet Radiation: Principles and Applications" (Cambridge University Press, 1999)

2. "Extreme ultraviolet microexposures at the Advanced Light Source using the 0.3 numerical aperture micro-exposure tool optic", Patrick P. Naulleau, Kenneth A. Goldberg, Erik Anderson, Jason P. Cain, Paul Denham, Keith Jackson, AnneSophie Morlens, Seno Rekawa, and Farhad Salmassi, J. Vac. Sci. Technol., B 22, 2962 (2004).

3. "Resist pattern prediction at EUV," John J. Biafore, Mark D. Smith, Eelco van Setten, Tom Wallow, Patrick Naulleau, David Blankenship, Stewart A. Robertson, and Yunfei Deng, Proc. SPIE, 7636, 76360R (2010).

4. "Pattern prediction in EUV resists," John J. Biafore, Mark D. Smith, Tom Wallow, Patrick Nalleau, David Blankenship, and Yunfei Deng, Proc. SPIE, 7520, 75201P (2009).
5. "Statistical simulation of photoresists at EUV and ArF," John J. Biafore, Mark D. Smith, Chris A Mack, James W. Thackeray, Roel Gronheid, Stewart A. Robertson, Trey Graves, and David Blankenship, Proc. SPIE, 7273, 727343 (2009).

6. (a.) "Chemically amplified resists resolving 25 nm 1:1 line: Space features with EUV lithography," James W. Thackeray, Roger A. Nassar, Robert Brainard, Dario Goldfarb, Thomas Wallow, Yayi Wei, Jeff Mackey, Patrick Naulleau, Bill Pierson, and Harun H. Solak, Proc. SPIE, 6517, 651719 (2007). (b) J. W. Thackeray, R. A. Nassar, K. Spear-Alfonso R. Brainard, D. Goldfarb, T. Wallow, Y. Wei, W. Montgomery, K. Petrillo, O. Wood, C. -S Koay, J. Mackey, P. Naulleau, B. Pierson, H. Solak, "Pathway to sub$30 \mathrm{~nm}$ Resolution in EUV Lithography, " $J$. Photopolym. Sci. Technol., 20 (3), pp. 411-418 (2007.

7. "Improved Lithographic Performance for EUV resists based on Polymers with Photoacid Generators in the Backbone," M. Thiyagarajan , K. Dean, K. Gonsalves, J. Photopolym. Sci. Technol., 18(6) pp. 737-741 (2005).

8. N. Turro, Modern Molecular Photochemistry], University Science Books, (1991).

9. "On a Heuristic Viewpoint Concerning the Production and Transformation of Light", A. Einstein, Annalen der Physik., 17, 1905.

10. "Parametric modeling of photoelectron effects in xray lithography", Ocola, L. E. J. Vac. Sci. Technol., B11 (1993).

11. "Modeling and simulation of chemicallyamplified electron beam, x-ray and EUV resist processes", T. Kozawa J. Vac. Sci. Technol., B22 (6), Nov/Dec 2004. 